\title{
ARTICLE Corticosterone after acute stress prevents the delayed effects on the amygdala
}

\author{
Prabahan Chakraborty $\mathbb{D D}^{1,5}$, Siddhartha Datta ${ }^{2}$, Bruce S. McEwen ${ }^{3}$ and Sumantra Chattarji $\mathbb{D}^{1,2,3,4,6}$
}

Even a single 2-hour episode of immobilization stress is known to trigger anxiety-like behavior and increase spine-density in the basolateral amygdala (BLA) of rats 10 days later. This delayed build-up of morphological and behavioral effects offers a stress-free time window of intervention after acute stress, which we used to test a protective role for glucocorticoids against stress. We observed that post-stress corticosterone, given 1 day after acute stress in drinking water, reversed enhanced anxiety-like behavior 10 days later. Quantification of spine-density on Golgi-stained BLA principal neurons showed that the same intervention also prevented the increase in spine numbers in the amygdala, at the same delayed time-point. Further, stress elevated serum corticosterone levels in rats that received vehicle in the drinking water. However, when stress was followed $24 \mathrm{~h}$ later by corticosterone in the drinking water, the surge in corticosterone was prevented. Together, these observations suggest that corticosterone, delivered through drinking water even $24 \mathrm{~h}$ after acute stress, is capable of reversing the delayed enhancing effects on BLA synaptic connectivity and anxiety-like behavior. Strikingly, although the immobilization-induced surge in corticosterone by itself has delayed detrimental effects on amygdalar structure and function, there exists a window of opportunity even after stress to mitigate its impact with a second surge of exogenously administered corticosterone. This provides a framework in the amygdala for analyzing how the initial physiological and endocrine processes triggered by traumatic stress eventually give rise to debilitating emotional symptoms, as well as the protective effects of glucocorticoids against their development.

Neuropsychopharmacology (2020) 45:2139-2146; https://doi.org/10.1038/s41386-020-0758-0

\section{INTRODUCTION}

Studies using animal models have identified features of stressinduced changes in amygdalar structure and function that are distinct from those seen in the hippocampus. Repeated stress, for example, causes loss of dendrites and spines in the rodent hippocampus [1, 2]. In the basolateral amygdala (BLA), by contrast, chronic stress strengthens the structural basis of synaptic connectivity through dendritic growth and increase in dendritic spines [1-3]. Further, these unique characteristics of stressinduced plasticity in the BLA are not restricted to chronic stress alone. For instance, a single 2-hour acute immobilization stress triggers a delayed onset of anxiety-like behavior and enhanced spine-density in the BLA not one, but ten days later [4-7].

This rodent model of a brief exposure to severe stress, triggering a delayed impact on the amygdala, is also interesting from a clinical perspective because it captures certain facets of post-traumatic stress disorder (PTSD). PTSD is triggered by an overwhelmingly traumatic, often life-threatening, event leading to prolonged changes in affective behavior $[8,9]$. Strikingly, some components of the fear response in PTSD last well beyond the original traumatic event and are accompanied by amygdalar hyperactivity. The present study draws upon clinical reports wherein cortisol treatment reduces the cardinal symptoms of
PTSD [10-14]. For instance, human subjects given cortisol infusions immediately after traumatic stress show a significant reduction of PTSD symptoms [14]. Early animal studies that explored this counterintuitive observation showed that corticosterone treatment $1 \mathrm{~h}$ after predator stress alleviated anxiety-like behavior on the elevated plus-maze [15] and reversed cellular and molecular effects on the hippocampus [14]. The amygdala has also been studied in this context - glucocorticoids protect against the delayed anxiogenic and cellular effects of acute stress - but using pre-stress corticosterone [5]. Corticosterone given $1 \mathrm{~h}$ before predator scent stress also prevents the delayed onset of PTSD-like symptoms in a rat strain with high basal anxiety [16]. However, will treatment with corticosterone after acute stress, which is clinically more relevant, be effective in reversing the delayed effects in the amygdala as well? If so, are the beneficial effects of such poststress interventions evident both at the cellular and behavioral levels? While corticosterone treatment immediately (1 h) after stress is effective in reversing changes in the hippocampus [14], will similar interventions after a significantly longer post-stress delay still work in the amygdala? Finally, while earlier studies focused primarily on testing exploration-based measures of anxiety-like behavior (e.g., elevated-plus maze), stress disorder patients also exhibit social anxiety as evidenced by enhanced gaze

\footnotetext{
${ }^{1}$ National Centre for Biological Sciences, Bangalore 560065, India; ${ }^{2}$ Centre for Brain Development and Repair, Institute for Stem Cell Biology and Regenerative Medicine, Bangalore 560065 , India; ${ }^{3}$ Laboratory of Neuroendocrinology, The Rockefeller University, New York, NY 10065, USA and ${ }^{4}$ Centre for Discovery Brain Sciences, Deanery of Biomedical Sciences, University of Edinburgh, Hugh Robson Building, 15 George Square, Edinburgh EH89XD, UK Correspondence: Sumantra Chattarji (shona@ncbs.res.in)

${ }^{5}$ Present address: Institut de Genomique Fonctionnelle, Inserm U1191, CNRS UMR5203, University of Montpellier, Montpellier 34090, France

${ }^{6}$ Present address: National Centre for Biological Sciences, GKVK Campus, Bellary Road, Bangalore, Karnataka 560065, India
}

Received: 15 April 2020 Revised: 5 June 2020 Accepted: 29 June 2020

Published online: 6 July 2020 
aversion [17]. Would post-stress glucocorticoid treatment also prevent social correlates of rodent anxiety?

Here we addressed these questions by testing whether supplementing corticosterone in drinking water $24 \mathrm{~h}$ after a single session of 2-h immobilization stress prevents the delayed increase, 10 days later, in both exploration and social interaction based measures of anxiety-like behavior. As morphological and behavioral effects of acute immobilization are not evident $24 \mathrm{~h}$ after stress [4], this time point offers a convenient post-stress time window for intervention before the onset of the more delayed changes. We also investigated the cellular correlates of these behavioral effects by probing if the same post-stress manipulation also prevents the delayed increase in BLA spinedensity.

\section{MATERIALS AND METHODS}

Animals

Male Wistar rats (50-60 days) were maintained on a $14 \mathrm{~h}: 10 \mathrm{~h}$ light: dark cycle, with ad libitum food and water. All maintenance and experimental procedures were approved by the Institutional Ethics Committee, National Centre for Biological Sciences, India.

\section{Stress}

Rats underwent a single episode of immobilization stress $(2 \mathrm{~h})$ (Fig. 1a), between $10 \mathrm{am}$ and $12 \mathrm{pm}$. Following this, they were returned to their home cages, and remained undisturbed until the day of experiment. Control rats, housed in a different room, were not stressed.

Elevated plus-maze

Rats were placed in the center of the elevated plus-maze with a free choice to explore the open ( 80 Lx) or closed arms ( $0 \mathrm{Lx})$ for 5 min [7]. Number of entries and time spent in the arms were analyzed offline, blind. Anxiety Index [5, 7] was computed using the following equation:

Anxiety Index $=1-\left[\left(\frac{\text { Open Arm Time }}{\text { Total Time }}+\frac{\text { Number of Open Arm Entries }}{\text { Total Entries }}\right) / 2\right]$

Juvenile social interaction test

Juvenile social interaction was carried out as described before $[18,19]$. Briefly, each adult rat freely interacted with a novel, unstressed juvenile male rat (28-36 days old) for $5 \mathrm{~min}$ on day 10 . Social exploration of the juvenile (classified into anogenital sniffing and head/torso exploration), aggressive behavior and exploratory rearing of the adult rat were quantified offline, blind.

Dendritic spine-density staining and analysis

Brains were stained by a modified Golgi-Cox technique [7, 20]. Slides were coded before morphological analysis, and the code was broken only after the analysis was completed. Spines were manually counted for a distance of $80 \mu \mathrm{m}$ from dendrite origin in BLA pyramidal neurons (between bregma $-2.16 \mathrm{~mm}$ to $-3.12 \mathrm{~mm}$ [21]), using Neurolucida (2017, Micro-BrightField, Williston, VT, USA) attached to an Olympus BX61 microscope (100x, 0.95 Numerical Aperture, Olympus BX61). All protrusions off the apical, primary dendrite were classified as spines, irrespective of their morphological characteristics. Imaging resolution being insufficient to resolve spine branching, each spine head was counted individually. A total of 7212 dendritic spines were manually analyzed from 130 neurons.

Corticosterone treatment

Habituation to the vehicle $(2.4 \%$ ethanol in drinking water, with two tablets of artificial sweetener per liter (Sugarfree, Zydus Wellness) to enhance palatability, $75 \mathrm{ml} /$ bottle, 2 bottles/ cage) in drinking water bottles started three days before stress. Subsequently, rats either received corticosterone (C2505, Sigma,
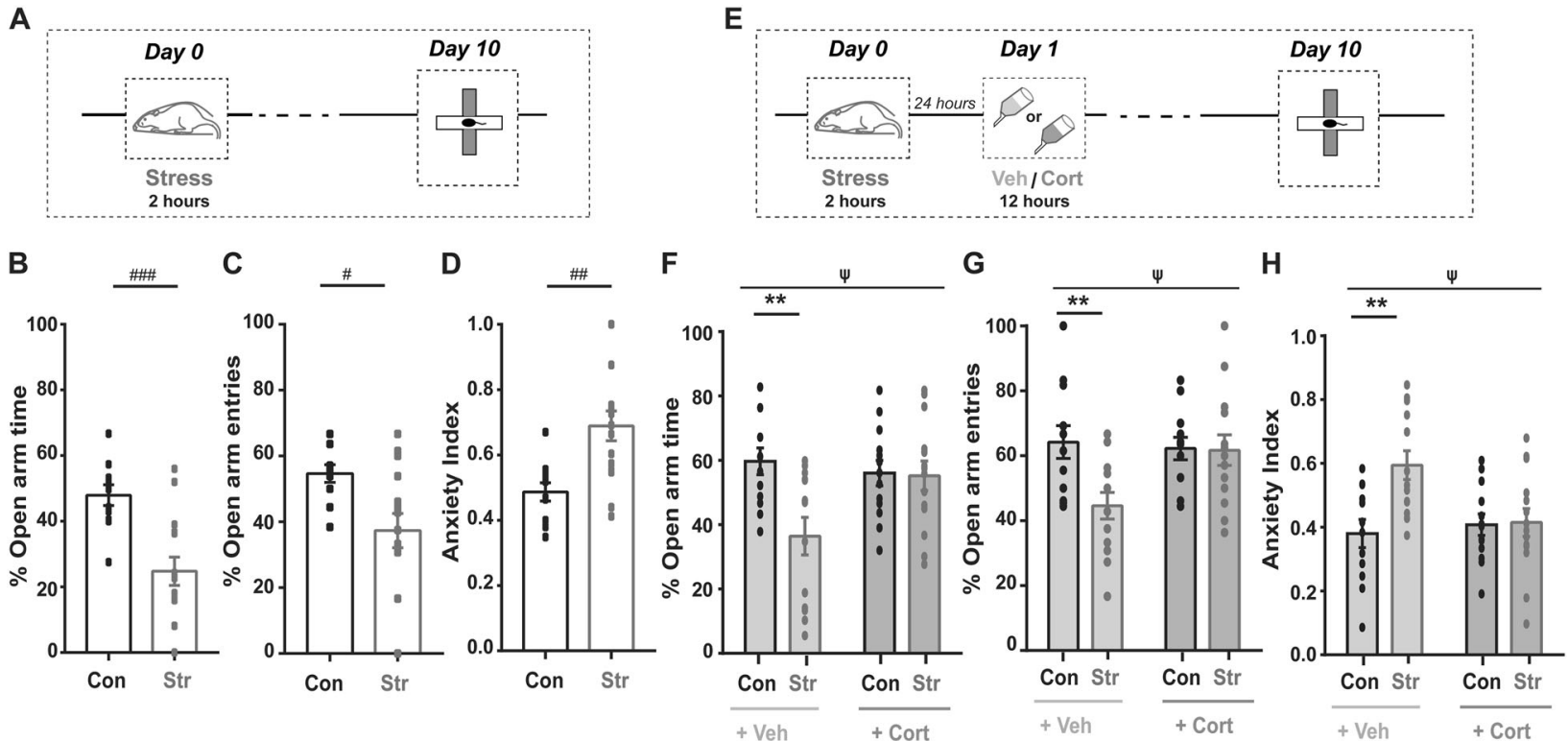

Fig. 1 Corticosterone in drinking water after stress prevents increase in anxiety-like behavior 10 days later. a Experimental design. Following $2 \mathrm{~h}$ of immobilization on day 0 , rats were subjected to elevated plus-maze 10 days later. b Stressed rats show reduced open arm time on the elevated plus-maze, 10 days after stress. c Stressed rats also show decreased open-arm entries. d Stressed rats show enhanced Anxiety Index on the elevated plus-maze, 10 days after stress. (Control, $N=11$ rats, Stress, $N=15$ rats). ${ }^{\#} p<0.05,{ }^{\# \#} p<0.01$ and ${ }^{\# \# \#} p<0.001$ in Unpaired t-test. e Experimental design. Rats received either corticosterone or vehicle in their drinking water bottle for $12 \mathrm{~h}$ (noon to midnight), $24 \mathrm{~h}$ after stress. Anxiety-like behavior was quantified 10 days later. $\mathbf{f}$ Post-stress corticosterone treatment, but not vehicle, prevented the decrease in open-arm time as well as (g) open-arm entries. $\mathbf{h}$ Corticosterone also prevents increased Anxiety Index, 10 days after stress. (Control + Vehicle, $N=25$ rats; Control + Corticosterone, $N=14$ rats; Stress + Vehicle, $N=30$ rats; Stress + Corticosterone, $N=14$ rats). ${ }^{\Psi} p<0.05$ in 'interaction' between factors stress and corticosterone, in Two-way ANOVA. ${ }^{* *} p<0.01$ in post-hoc Sidak's test. 
$400 \mu \mathrm{g} / \mathrm{ml}$ in vehicle) $24 \mathrm{~h}$ after stress (12 p.m. - 12 a.m., day 2$)$, or remained on vehicle. Dosage and treatment duration was adapted from a previous report found to elicit protective effects on stressinduced amygdalar plasticity [5]. At midnight, day 2, regular drinking water was reintroduced until day 10.

\section{Corticosterone ELISA}

Trunk blood collected from different cohorts of rats were centrifuged at $4{ }^{\circ} \mathrm{C}$, and serum was separately stored at $-20^{\circ} \mathrm{C}$ until analysis. Corticosterone ELISA was performed as previously described [7] following kit instructions (Enzo Life Sciences, ADI900-097).

\section{Statistical analysis}

All values are reported as Mean \pm SEM. Data sets were evaluated for outliers (greater than twice the standard deviation away from the mean). Unpaired two-tailed t-test and ordinary or repeated-measures two-way ANOVA, followed by post-hoc Sidak's test or Tukey's test, were used as and when applicable. All statistical analyses and plots were computed using GraphPad Prism (GraphPad software Inc., La Jolla, California, USA, version 6). Please see Table S1 for detailed description of statistical results.

\section{RESULTS}

Oral administration of corticosterone $24 \mathrm{~h}$ after acute stress prevents the delayed anxiogenic effects

Before testing the efficacy of post-stress interventions, we first wanted to confirm the delayed anxiogenic effects of 2-hour acute immobilization stress (Fig. 1a). Indeed, this was manifested as a reduction in both the percentage of time spent in the open-arm (Fig. 1b; Control, Con: $47.95 \pm 3.1 \%, N=11$; Stress, Str: $24.8 \pm 4.3 \%$, Con, Str, $N=15$ ) as well as percentage of open-arm entries, 10 days after stress (Fig. 1c; Con: $54.7 \pm 2.67 \%$, Str: $37.4 \pm 5.2 \%$ ). Consequently, the anxiety index (Methods) was significantly higher in stressed rats (Fig. 1d; Con: $0.49 \pm 0.03$, Str: $0.69 \pm 0.05$ ). Total number of entries was not affected by stress, ruling out any impairment in locomotor activity (Fig. S1b) [22]. These results are consistent with previous reports $[4-7,23]$.

In light of earlier clinical studies [11, 12, 14, 24], we next tested if corticosterone treatment after acute stress prevents its delayed anxiogenic effects. To avoid injection and oral gavage which are known to be stressful and elevate corticosterone levels in rodents and in turn have been shown to prevent the behavioral and cellular effects of stress [5, 7], we administered either corticosterone or vehicle through drinking water $24 \mathrm{~h}$ after acute immobilization stress. Post-stress treatment lasted for $12 \mathrm{~h}$ (noon to midnight) and its behavioral effects were assessed on the plusmaze 10 days after stress (Fig. 1e).

First, vehicle alone did not prevent the delayed anxiogenic effects, as vehicle-treated stressed rats showed reductions in both percentage open-arm time (Fig. 1f; Control + Vehicle, Con-Veh: $58.6 \pm 3.2 \%, N=12$, Stress + Vehicle, Str-Veh: $37.4 \pm 4.1 \%, N=13$ ) and entries (Fig. 1g; Con-Veh: 64.2 $\pm 5.0 \%$, Str-Veh: $44.6 \pm 4.1 \%$ ), resulting in a higher anxiety index (Fig. $1 \mathrm{~h}$; Con-Veh: $0.39 \pm 0.03$, Str-Veh: $0.60 \pm 0.04)$. In striking contrast, corticosterone administered $24 \mathrm{~h}$ after acute stress prevented these anxiogenic effects. Percentage open-arm time (Fig. 1f, Control + Corticosterone, ConCort: $55.2 \pm 4.6 \%, N=13$, Stress + Corticosterone, Str-Cort: $37.4 \pm$ $4.1 \%, N=14$ ), entries (Fig. 1g, Con-Cort: $60.2 \pm 3.95 \%$, Str-Cort: 61.7 $\pm 4.7 \%$ ) and anxiety index (Fig. $1 \mathrm{~h}$; Con-Cort: $0.42 \pm 0.04$, Str-Cort: $0.41 \pm 0.03$ ) in corticosterone-treated stressed rats were all comparable to their control counterparts. A two-way ANOVA revealed significant interaction between the factors 'stress' and 'corticosterone', implying that corticosterone treatment prevented stress-induced increase in anxiety-like behavior on the plus-maze. Total number of entries remained unaffected (Fig. S1d), ruling out any differences in locomotor activity between experimental groups.

Oral administration of corticosterone $24 \mathrm{~h}$ after stress prevents delayed impairment in social interactions

Could the protective effects of corticosterone treatment extend to delayed manifestations of social anxiety? To address this question, we used a previously established juvenile social interaction procedure $[18,19]$ involving exploration of a juvenile conspecific by an adult subject (Fig. 2a). A significant advantage of this paradigm over conventional social interaction tests on adult rats lies in the fact that the same juvenile rat can be used for multiple trials without any experimental confounds [18, 19]. Furthermore, both rats interact freely in a simple experimental arena, thereby increasing the test's ethological relevance. We observed that 10 days later, stressed adult rats spent significantly less time interacting with a juvenile (Fig. 2b; Con: $158.3 \pm 7.0$ s, $N=19$; Str: $125.70 \pm 6.9 \mathrm{~s}, N=24)$. A more detailed assessment of the two components that constituted social interaction behavior revealed a significant stress-induced decrease in the time spent in anogenital sniffing (Fig. 2c; Con: $94.6 \pm 8.1 \mathrm{~s}$; Str: $54.1 \pm 5.4 \mathrm{~s}$; $\mathrm{t}_{41}=4.28$ ), but not head/torso sniffing (Fig. S2b). Further, stress did not alter exploratory rearing, an asocial measure of anxiety (Fig. S2c). Finally, stress exposure did not alter the time spent in aggressive behaviors towards juveniles (Fig. S3a), which only constituted a very small fraction $(2-3 \%)$ of the total behavioral repertoire exhibited by the rats (Fig. S3b). Together, these indicate that acute stress leads to a specific impairment in the anogenital component of social interaction 10 days later, without any effects on exploratory rearing or aggression.

Does corticosterone treatment $24 \mathrm{~h}$ after acute stress also reverse the delayed deficit in social interaction (Fig. $2 \mathrm{~d}$ )? We found that vehicle treatment alone could neither prevent the delayed decrease in social interaction (Fig. 2e; Con-Veh: $159.9 \pm 7.97 \mathrm{~s}, N=$ 16, Str-Veh: $130.8 \pm 6.8 \mathrm{~s}, N=16$ ), nor in anogenital sniffing behavior (Fig. 2f; Con-Veh: $93.24 \pm 8.1 \mathrm{~s}$, Str-Veh: $63.95 \pm 5.0 \mathrm{~s}$ ). However, corticosterone treatment showed a reversal of both time spent in social interaction (Fig. 2e; Con-Cort: $135.8 \pm 5.7 \mathrm{~s}, N=15$, Str-Cort: $156.4 \pm 7.5 \mathrm{~s}, N=18$ ), as well as time spent in anogenital sniffing (Fig. 2f; Con-Cort: $73.9 \pm 6.5 \mathrm{~s}$, Str-Cort: $90.9 \pm 5.7 \mathrm{~s}$ ). Statistical analysis further revealed a significant interaction between factors 'stress' and 'corticosterone'. Head/torso sniffing (Fig. S2e) and exploratory rearing (Fig. S2f) remained unaltered across all groups.

Post-stress corticosterone also prevents the delayed increase in spine-density in the BLA

Our finding that post-stress corticosterone reverses the delayed anxiogenic effects suggest that despite two successive manipulations that both increase corticosterone levels, BLA neurons in these rats should not undergo stress-induced delayed morphological plasticity. Thus, we next tested if corticosterone treatment $24 \mathrm{~h}$ after stress also prevents such a stress-induced increase in the BLA (Fig. 3a). First, 10 days after stress, vehicle-treated rats showed an increase in spine-density along primary apical dendrites of BLA principal neurons (Fig. 3c, d; Con-Veh: $50.5 \pm 3.2, N=6$, Str-Veh: $62.3 \pm 3.5, n=7)$ that approached statistical significance $(p=0.05)$. On the other hand, corticosterone administered through drinking water $24 \mathrm{~h}$ after stress reversed this effect 10 days later (Fig. 3b, c; Con-Cort: $58.1 \pm 3.3, N=6$, Str-Cort: $48.3 \pm 2.5, N=7$ ). This was evident as a statistically significant interaction between the factors 'stress' and 'corticosterone' in a two-way ANOVA, confirming a change in stress-induced increase in spine-density due to corticosterone treatment.

The combined findings on the behavioral and morphological effects of acute immobilization stress, and their reversal using post-stress corticosterone, are summarized in Fig. 3d. Vehicletreated stressed rats (Fig. $3 \mathrm{~d}$, red circle) exhibit higher anxiety 
A

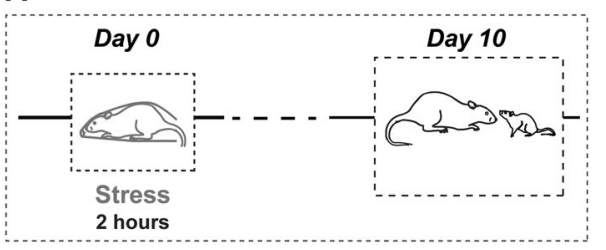

B

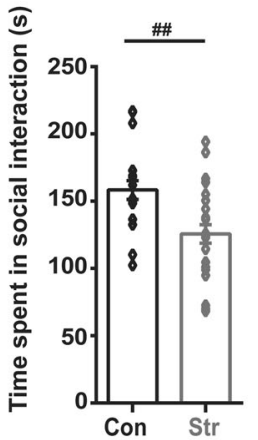

C
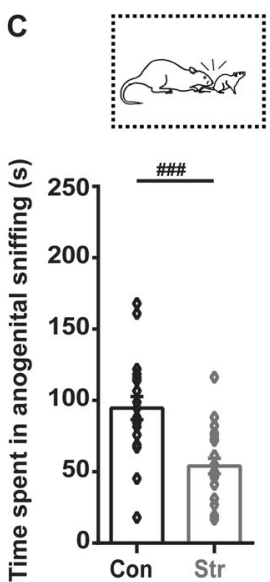

D

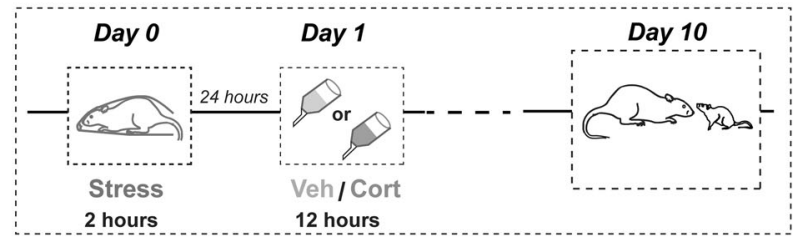

E

$\mathbf{F}$
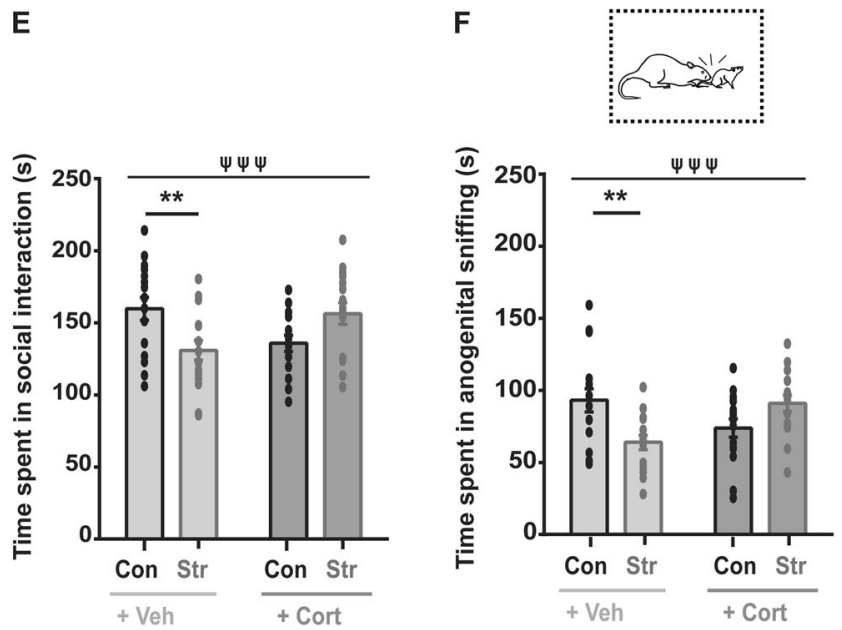

Fig. 2 Corticosterone in drinking water after stress prevents impairment in social interaction with a juvenile rat 10 days later. a Experimental design. Following acute immobilization stress on day 0 , rats underwent social interaction with a male, unfamiliar juvenile rat, 10 days later. b Stressed rats spend significantly less time interacting with a juvenile conspecific. c Stress leads to a significant impairment in anogenital exploration of a juvenile. (Control, $N=19$ rats, Stress, $N=24$ rats). ${ }^{\# \#} p<0.01$ and ${ }^{\# \# \# ~} p<0.001$ in Unpaired t-test. d Experimental design. Rats received either corticosterone or vehicle in their drinking water bottle for $12 \mathrm{~h}$ (noon to midnight), $24 \mathrm{~h}$ after stress. Social interaction with a male, unfamiliar juvenile rat was quantified 10 days later. e Vehicle-treated stressed rats show a significant reduction in total social interaction, which is rescued by post-stress corticosterone. f Post-stress corticosterone significantly prevents decreased anogenital exploration of a juvenile conspecific. (Control + Vehicle, $N=16$ rats; Control + Corticosterone, $N=15$ rats; Stress + Vehicle, $N=18$ rats; Stress + Corticosterone, $N=16$ rats). ${ }^{\Psi} \Psi^{\prime}{ }_{p}<0.001$ in 'interaction' between factors stress and corticosterone, in Two-way ANOVA. ${ }^{* *} p<0.01$ in post-hoc Sidak's test.

indices (Anxiety, upward arrow) and elevated levels of BLA spine-density (Spine-density, rightward arrow), compared to their control counterparts (Fig. 3d, black circle). On the otherhand, corticosterone treatment after stress brings spine-density and anxiety indices closer to control levels, although these rats experienced two consecutive surges in corticosterone levels $24 \mathrm{~h}$ apart. Thus, high and low levels of BLA spine-density were accompanied by high and low anxiety indices respectively, although they resulted from rather different conditions.

Post-stress corticosterone prevents stress-induced elevation in corticosterone

In both humans and rodents, exposure to stress leads to enhanced systemic levels of cortisol and corticosterone respectively, that last from minutes to days afterward [25-27]. This stress-induced surge triggers plasticity mechanisms that eventually culminate in persistent structural and functional changes in the brain [3]. It is in this context that our findings are particularly puzzling because two successive manipulations that both elevate corticosterone levels (acute stress and corticosterone in the drinking water), end up preventing rather than adding up to elicit even bigger cellular and behavioral effects 10 days later. Could it be that the initial surge in corticosterone elicited by the immobilization stress is somehow dampened by the subsequent corticosterone treatment, thereby reversing its eventual cellular and behavioral effects? We examined this possibility by measuring serum corticosterone levels on Day 2, $12 \mathrm{~h}$ after the end of corticosterone treatment via drinking water (Day 1) and $48 \mathrm{~h}$ after the end of acute stress on
Day 0 (Fig. 4a). In vehicle-treated rats, stress-triggered high levels of corticosterone relative to vehicle- and corticosterone-treated unstressed controls (Fig. 4b; Con-Veh: $12.3 \pm 2.8 \mathrm{ng} / \mathrm{ml}, N=7$, StrVeh: $56.7 \pm 6.7 \mathrm{ng} / \mathrm{ml}, \quad N=4$, Con-Cort: $5.9 \pm 1.1 \mathrm{ng} / \mathrm{ml}, \quad N=8$ ). However, such an increase was prevented in stressed rats that received corticosterone $24 \mathrm{~h}$ after stress (Day 1, Fig. 4b; Str-Cort: $16.3 \pm 2.6 \mathrm{ng} / \mathrm{ml}, N=5$ ), whose levels were significantly lower than vehicle-treated stressed rats, and comparable to vehicle-treated controls. Effects of both stress, corticosterone as well as their interaction were significant in a two-way ANOVA. Thus, instead of the two surges of corticosterone adding up, post-stress treatment reversed the initial surge of corticosterone elicited by stress. And in these rats, the delayed BLA spine-density increase and anxiogenic effects were blocked.

Finally, we wanted to ensure that consumption of corticosterone and vehicle were similar across all experimental groups. As rats had free access to drinking water containing either vehicle or corticosterone, we quantified volume consumed per cage once every $4 \mathrm{~h}$ during the 12 -h treatment period (Fig. $4 \mathrm{c}$ ). Notably, there was no difference in the volume of water consumed between stress and control rats treated with either vehicle or corticosterone, and they showed equivalent patterns of consumption throughout the duration (Fig. 4d, e, Table S2). But was oral delivery successful in raising systemic levels of corticosterone? Control rats receiving corticosterone for $12 \mathrm{~h}$ (Fig. 4f) showed a greater increase in systemic levels as compared to vehicle-treated controls (Fig. 4g; Con-Veh: $18.10 \pm 6.14 \mathrm{ng} / \mathrm{ml}, \quad N=8$, Con-Cort: $101.45 \pm 14.36 \mathrm{ng} / \mathrm{ml}, N=5$ ), thereby confirming the efficacy of the treatment protocol used here. 
A

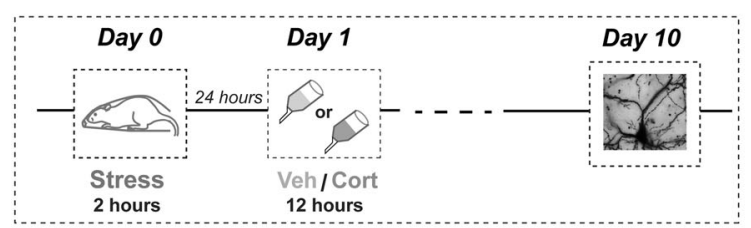

D

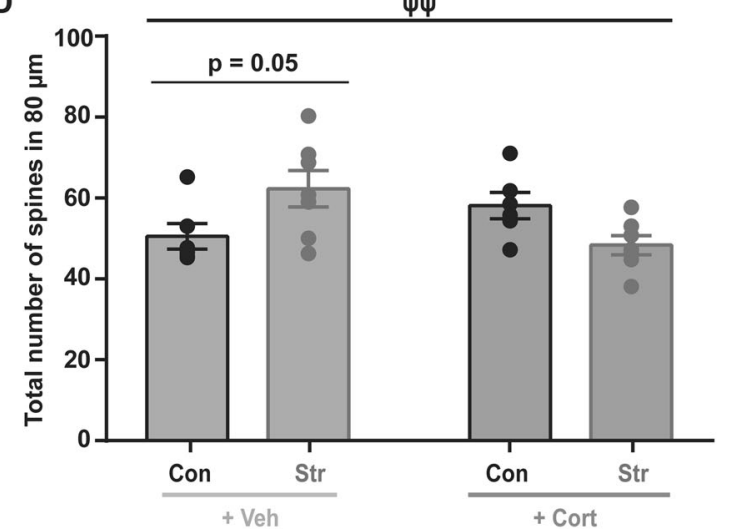

B

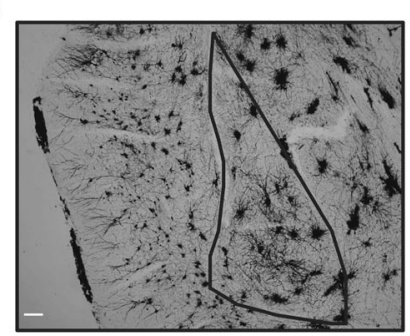

E

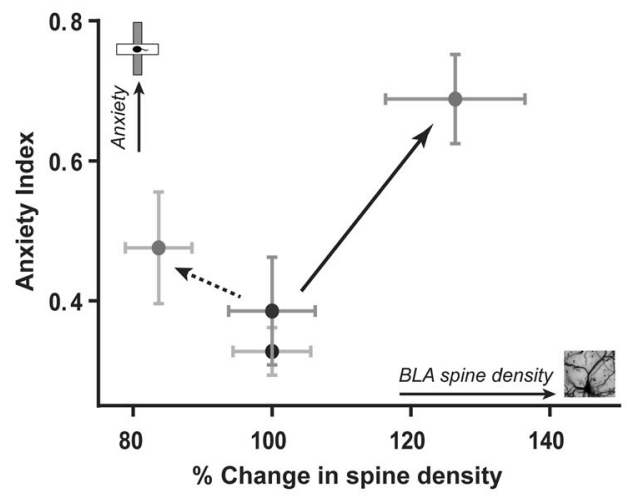

C

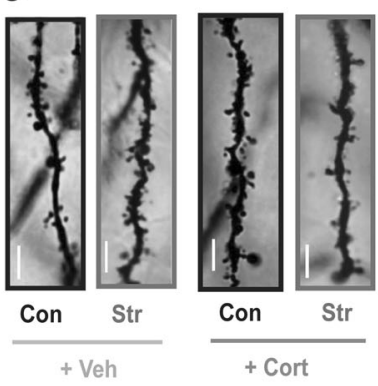

- Con + Veh

- Str + Veh

- Con + Cort

- Str + Cort

Fig. 3 Post-stress corticosterone prevents the delayed increase in spine-density in the basolateral amygdala (BLA). a Experimental design. Rats received either corticosterone or vehicle in their drinking water bottle for $12 \mathrm{~h}$ (noon to midnight), 1 day after stress. Spine-density in the BLA were quantified 10 days later. b Representative image showing a Golgi-stained coronal section of a rat BLA. Scale bar $200 \mu$ m. c Representative images of dendritic spines from the experimental groups. Scale bar $10 \mu \mathrm{m}$. d Post-stress corticosterone, but not vehicle, also prevented elevated spine-density in the BLA. (Control + Vehicle, $N=6$ rats; Control + Corticosterone, $N=6$ rats; Stress + Vehicle, $N=7$ rats; Stress + Corticosterone, $N=7$ rats). ${ }^{\Psi} \Psi p<0.01$ in 'interaction' between factors stress and corticosterone, in Two-way ANOVA. e Percentage change in BLA spine-density, normalized to respective controls, has been plotted along the $x$-axis and change in Anxiety Index of the same rats along the $y$-axis. While vehicle-treated stressed rats showed an increase in both spine-density and anxiety (diagonal solid arrow), these large changes in both cellular and behavioral measures was not seen with post-stress corticosterone treatment (diagonal dashed arrow). (Control + Vehicle, $N=6$ rats, Stress + Vehicle, $N=7$ rats, Control + Corticosterone, $N=6$ rats, Stress + Corticosterone, $N=7$ rats).

\section{DISCUSSION}

Here our goal was to explore the possibility of reversing the delayed effects of stress through interventions after rats have already experienced stress. The choice of post-stress intervention was inspired by somewhat counterintuitive clinical findings that administration of cortisol after victims have experienced trauma reduces the eventual development of the cardinal symptoms of PTSD $[10,12-14,28,29]$. To this end, we built upon earlier studies that showed a 2-hour episode of acute immobilization stress in rats enhances anxiety-like behavior on the elevated plus-maze and triggers increase in BLA spine-density 10 days later $[4-7,23,30]$. These delayed effects offered a post-stress time window for administering corticosterone in the drinking water $24 \mathrm{~h}$ after subjecting rats to stress. This treatment reversed both the cellular and behavioral effects of acute stress 10 days later (Fig. 4h). First, oral administration of corticosterone after stress prevented the reduction in open-arm exploration on the elevated plus-maze. Next, we extended this analysis to also examine the impact of the same stressor on social measures of anxiety-like behavior. Stressed rats exhibited impairment in social interaction with a juvenile conspecific 10 days later, and this deficit too was rescued by post-stress corticosterone. Second, in light of accumulating evidence that the delayed anxiogenic effects of acute stress is paralleled by delayed morphological changes in BLA principal neurons, our behavioral results led us to test the efficacy of the same post-stress corticosterone treatment at the cellular level. Indeed, the same treatment protected against stressinduced increase in the density of dendritic spines 10 days later. Finally, although both groups of rats experienced the same 2-hour immobilization, there was one key difference- rats subjected to stress followed by oral corticosterone did not undergo the increase in serum corticosterone levels (bottom, Fig. 4h) that was seen in the other group receiving only vehicle after stress (top, Fig. 4h). Taken together, these results suggest that the natural surge in corticosterone elicited by immobilization stress can be reversed by a subsequent exogenous treatment of corticosterone, as late as a day after acute stress, which in turn prevented the delayed detrimental effects at the behavioral and cellular levels.

Thus, consistent with clinical reports, these findings add to growing evidence from animal studies on the protective effects of glucocorticoids against the delayed impact of stress across levels of neural organization $[14,15,31-36]$. In an earlier study, we used the same model of acute immobilization to demonstrate that elevating corticosterone levels before stress prevents the delayed effects on the BLA [5]. A subsequent study showed a similar protective effect of oral gavage of vehicle given $1 \mathrm{~h}$ after immobilization - raising the possibility that a surge in corticosterone elicited by the gavage procedure dampened the delayed detrimental effects [7]. The present study is motivated by, and extends, this earlier observation. First, building upon the protective effects of the stress caused by oral gavage, we have now used a strategy that directly tests the role of corticosterone, which is also relevant in light of earlier clinical reports [10, 12$14,24]$. Second, we have done this by significantly extending the window of intervention well beyond $1 \mathrm{~h}$ to $24 \mathrm{~h}$ after the end of stress. Future studies will be needed to explore how long this post-stress window of effective intervention lasts. We have also expanded our analysis to social behaviors, thereby testing the 
A

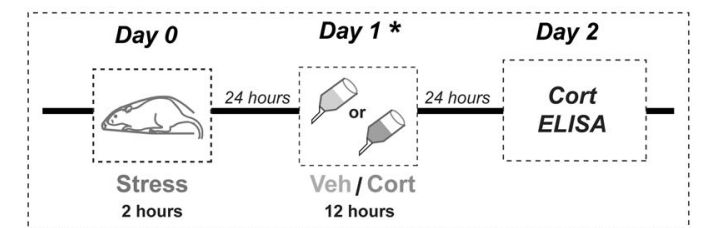

B

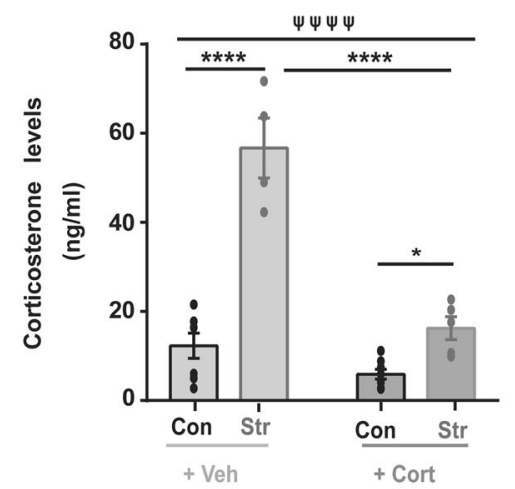

$\mathbf{F}$

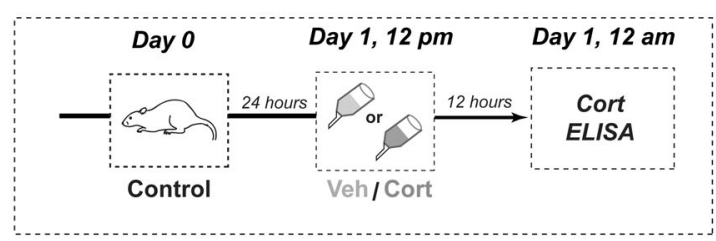

G

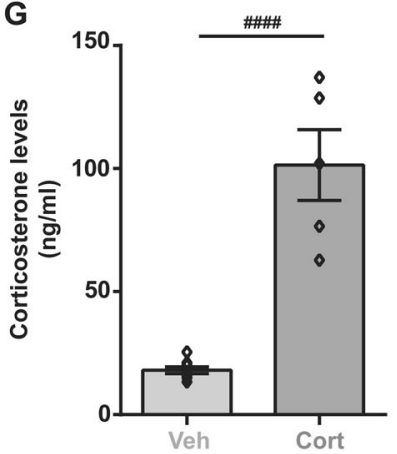

C

* Day 1

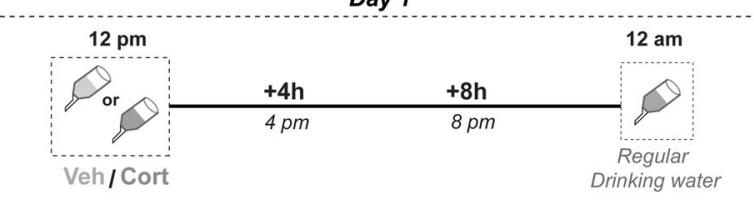

D

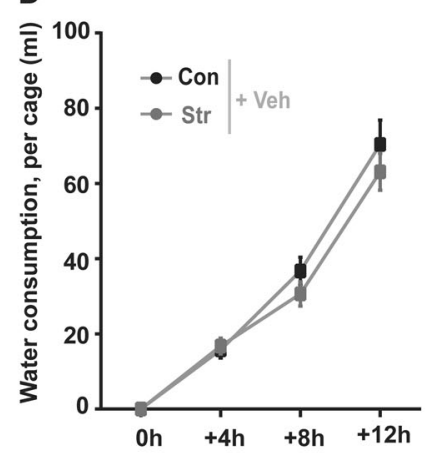

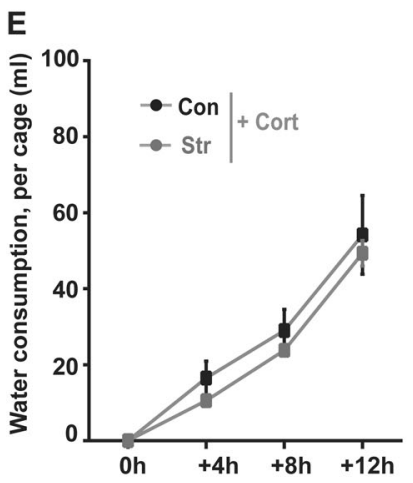

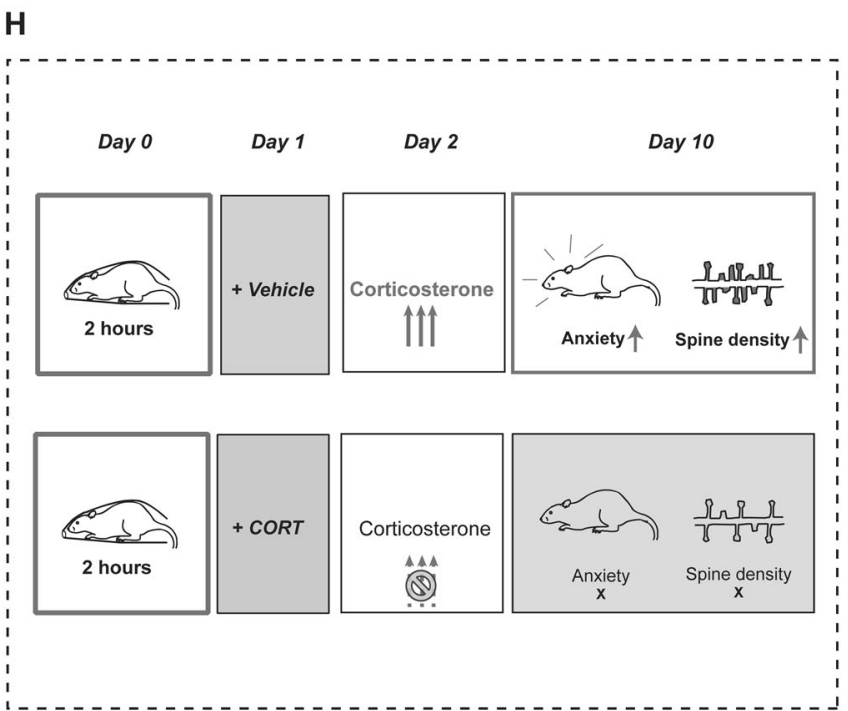

Fig. 4 Post-stress corticosterone treatment leads to a reduction in stress-induced increase in serum corticosterone levels. a Following $2 \mathrm{~h}$ of immobilization stress (day 0 ), rats received either corticosterone or vehicle in their drinking water bottle $24 \mathrm{~h}$ later (day 1 ). Treatment was carried out for a period of $12 \mathrm{~h}$ (noon to midnight). $12 \mathrm{~h}$ after the end of corticosterone treatment, rats were sacrificed on day 2 and serum levels of corticosterone were quantified with an ELISA. b Day 2: Vehicle-treated stressed rats showed significantly elevated levels of serum corticosterone. In comparison, corticosterone treatment causes a significant reduction in serum corticosterone on day 1 , although levels are higher than corticosterone-treated control rats. (Control + Vehicle, $N=7$ rats; Control + Corticosterone, $N=8$ rats; Stress + Vehicle, $N=4$ rats; Stress + Corticosterone, $N=5$ rats). ${ }^{\Psi}{ }^{\top} \Psi^{\Psi} \Psi_{p}<0.0001$ for 'interaction' between factors stress and corticosterone, in Two-way ANOVA. ${ }^{* * * *} p<$ 0.0001 and ${ }^{*} p<0.05$ in post-hoc Sidak's test. c Day 1: Consumption of drinking water containing vehicle or corticosterone was measured per cage every $4 \mathrm{~h}$ between noon and midnight. Regular drinking water was replaced at the end of the treatment period. $\mathbf{d}$ Cumulative plot of consumption over time show that stressed and control rats consumed vehicle and e corticosterone to the same extent over the treatment period (Control + Vehicle, $N=8$ cages; Control + Corticosterone, $N=6$ cages; Stress + Vehicle, $N=9$ cages; Stress + Corticosterone, $N=9$ cages). f To quantify elevation in systemic levels of corticosterone, control rats were sacrificed at midnight after consumption of either vehicle or corticosterone during the treatment period. $g$ Corticosterone treatment causes a significant elevation of serum levels of the glucocorticoid, in

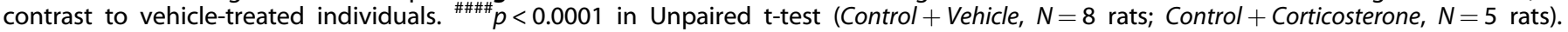
h Summary of findings. (Top) Stressed rats treated with vehicle $24 \mathrm{~h}$ after stress show elevated levels of corticosterone, which is followed by enhanced anxiety-like behavior and elevated spine-density in the BLA 10 days later. (Bottom) By contrast, when corticosterone is administered $24 \mathrm{~h}$ after stress, this prevents the increase in corticosterone, and also the delayed increase in anxiety-like behavior and BLA spine-density 10 days later. CORT Corticosterone.

efficacy of this intervention on different aspects of anxiety-like behavior affected by stress. Interestingly, oral administration caused a significant elevation of systemic corticosterone that was higher than vehicle treatment for the same time. It was also higher than a previously reported post-immobilization surge [7]. High doses of post-stress glucocorticoids have indeed been found to be protective against stress, in agreement with this finding [14]. Importantly, earlier studies have also examined changes in the hippocampus in the context of post-stress interventions. For instance, administration of hydrocortisone $1 \mathrm{~h}$ after predator scent stress blocked the delayed increase in anxiety, as well as dendritic spine loss in the dorsal dentate gyrus [14]. In light of the present 
findings in the amygdala, it will be interesting to explore if the time window of post-stress intervention can be extended beyond $1 \mathrm{~h}$ for reversing stress-induced structural plasticity in the dentate gyrus and other hippocampal sub-regions that undergo stressinduced modifications [2]. It is also interesting to note that corticosterone treatment led to no observable effects on spinedensity or anxiety-like behavior in control rats. This is in agreement with a previous report, where treatment with the same dosage of corticosterone for $12 \mathrm{~h}$ also did not have cellular and behavioral effects [5]. In contrast, acute corticosterone injections have been shown to cause delayed dendritic hypertrophy of BLA neurons and increase anxiety-like behavior [37, 38], which may be due to differences in the mode of delivery (i.p. injection versus drinking water) and the measure of morphological plasticity (dendrites versus spines). It is also possible that oral corticosterone, as well as stress, alters dendritic spine turnover, formation of new spines, spine involution and dynamic changes in their shape and maturity. An assessment of such micro-structural changes could reveal finer aspects of the regulation of spine plasticity in the amygdala with stress and corticosterone that have not been explored here [39].

While the present study used an animal model to explore mechanistic underpinnings of the protective effects of glucocorticoids against the delayed effects of stress [34-36], there are also reports suggesting either insufficient evidence or only a limited role for hydrocortisone in preventing the development of PTSD [40, 41]. Another potential limitation of this approach stems from the reported side-effects of glucocorticoid treatment [42], underscoring the need to exercise caution in using this strategy in susceptible individuals. On the other hand, treating stress with glucocorticoids also resembles stress inoculation, wherein prior exposure to brief stressors helps to cope with futures episodes of stress [43]. As we have reported previously, pretreatment with glucocorticoids indeed has a protective effect on the delayed effects acute immobilization [5]. Protective effects of stress inoculation has been observed in other species too, including humans [44-46]. The results reported here also provide a useful framework for probing this, by testing if stress and corticosterone exposure together confer resilience to future episodes of stress exposure.

Finally, perhaps the most striking observation from the present study is that two successive manipulations that both, on their own, increase corticosterone levels, together bring back anxiety and BLA spine-density to unstressed levels. Hence, future analysis of the potential mechanisms underlying this will have to focus on the signaling mechanisms triggered by acute stress and corticosterone treatment alone, and the interplay between them. Recent studies point to several lines of evidence that may be useful in this regard. For instance, presynaptic release of glutamate is enhanced immediately [30], as well as 10 days after acute immobilization stress [6]. Notably, acute immobilization stress also causes an immediate decrease in synaptic inhibition in BLA neurons [30]. This synaptic disinhibition, combined with elevated levels of glutamate during and soon after acute stress, is likely to enhance the excitability of BLA principal neurons. Further, N-methyl-D-aspartate (NMDA) receptors in the BLA during acute stress play a role in the delayed formation of dendritic spines [6]. Taken together, this suggests that higher glutamate levels, by activating NMDA receptors in the BLA, could create an ideal synaptic substrate for the delayed increase in dendritic spines. Therefore, it would be of interest to examine if in vivo corticosterone treatment exerts its protective effects by acting upon similar receptor mechanisms and signaling pathways in the BLA. Multiple lines of evidence also suggest a possible role of glucocorticoid receptor (GR)-dependent signaling mechanisms. On one hand, GRagonist dexamethasone enhances fear extinction by dosedependently modulating methylation of the GR-chaperone FKBP5 in the BLA $[47,48]$. Genetic polymorphisms of FKBP5 associate with PTSD symptoms [47], suggesting its involvement both in the effects of stress, as well as their prevention. On the other hand, GRs also regulate metaplasticity in the amygdala which, for instance, underlies suppression of fear memory recall by prior exposure to acute stress [49]. Mechanistically, while acute restraint alone increases the excitatory activity of BLA principal neurons, treating these cells ex vivo with an additional dose of corticosterone prevents stress-enhanced glutamate release by GR-mediated activation of endocannabinoid signaling $[50,51]$. A recent study also identified a role of endocannabinoids in the delayed strengthening of BLA synaptic connectivity after acute immobilization [30]. It remains to be seen if the two surges of corticosterone (caused by immobilization, followed by corticosterone treatment) is able to mitigate the delayed effects of this stressor through similar cellular mechanisms.

In conclusion, our results also point to future directions of enquiry that are of clinical relevance. For example, how long does the post-stress intervention window extend beyond the 24-h period reported here? And, how long lasting are these protective effects of corticosterone? The findings presented here offer an experimental framework, spanning multiple levels of neural organization, for elucidating the structural and functional alterations triggered by traumatic stress over time, and their reversal using clinically effective therapeutic interventions such as glucocorticoid treatment.

\section{FUNDING AND DISCLOSURE}

This work was supported by funds from the Tata Institute of Fundamental Research, Department of Atomic Energy and Department of Biotechnology, Government of India. The authors declare no financial interests or potential conflicts of interest.

\section{ACKNOWLEDGEMENTS}

PC and SC would like to express their unending gratitude to Prof. Bruce McEwen for his generous advice and continued guidance during this project, from the early days of its conceptualization to its completion.

\section{AUTHOR CONTRIBUTIONS}

PC, BM and SC contributed to the experimental design. PC and SD performed the experiments and analyzed the data. PC, BM and SC interpreted the results. PC and SC wrote the manuscript.

\section{ADDITIONAL INFORMATION}

Supplementary Information accompanies this paper at (https://doi.org/10.1038/ s41386-020-0758-0)

Publisher's note Springer Nature remains neutral with regard to jurisdictional claims in published maps and institutional affiliations.

\section{REFERENCES}

1. Chattarji AS, Tomar A, Suvrathan A, Ghosh S. Neighborhood matters: divergent patterns of stress - induced plasticity across the brain Affiliations. Nat Publ Gr. 2015;18:1-39.

2. McEwen BS, Nasca C, Gray JD. Stress effects on neuronal structure: hippocampus, amygdala and prefrontal cortex. Neuropsychopharmacology. 2016;41:3-23. https://doi.org/10.1038/npp.2015.171.

3. Roozendaal B, McEwen BS, Chattarji S. Stress, memory and the amygdala. Nat Rev Neurosci. 2009;10:423-33.

4. Mitra R, Jadhav S, McEwen BS, Vyas A, Chattarji S. Stress duration modulates the spatiotemporal patterns of spine formation in the basolateral amygdala. Proc Natl Acad Sci USA. 2005;102:9371-6.

5. Rao RP, Anilkumar S, McEwen BS, Chattarji S. Glucocorticoids protect against the delayed behavioral and cellular effects of acute stress on the amygdala. Biol Psychiatry. 2012;72:466-75. 
6. Yasmin F, Saxena K, McEwen BS, Chattarji S. The delayed strengthening of synaptic connectivity in the amygdala depends on NMDA receptor activation during acute stress. Physiol Rep. 2016;4:1-11.

7. Chakraborty $P$, Chattarji S. Interventions after acute stress prevent its delayed effects on the amygdala. Neurobiol Stress. 2019;10:1-23.

8. Rao RP, Suvrathan A, Miller MM, McEwen BS, Chattarji S. PTSD: From Neurons to Networks. Neurobiol. PTSD. New York: Humana Press; 2009. p. 151-85.

9. Bryant RA. Post-traumatic stress disorder: a state-of-the-art review of evidence and challenges. World Psychiatry. 2019;18:259-69. https://doi.org/10.1002/wps.20656.

10. Schelling G, Kilger E, Roozendaal B, De Quervain DJF, Briegel J, Dagge A, et al. Stress doses of hydrocortisone, traumatic memories, and symptoms of posttraumatic stress disorder in patients after cardiac surgery: A randomized study. Biol Psychiatry. 2004;55:627-33.

11. Weis F, Kilger E, Roozendaal B, De Quervain DJF, Lamm P, Schmidt M, et al. Stress doses of hydrocortisone reduce chronic stress symptoms and improve healthrelated quality of life in high-risk patients after cardiac surgery: a randomized study. J Thorac Cardiovasc Surg. 2006;131:277-82.

12. Schelling G, Roozendaal B, Krauseneck T, Schmoelz M, De Quervain D, Briegel J. Efficacy of hydrocortisone in preventing posttraumatic stress disorder following critical illness and major surgery. Ann N Y Acad Sci. 2006;1071:46-53.

13. Schelling G, Briegel J, Roozendaal B, Stoll C, Rothenha $H$. The effect of stress doses of hydrocortisone during septic shock on posttraumatic stress disorder in survivors. Biol Psychiatry. 2001;50:978-85.

14. Zohar J, Yahalom H, Kozlovsky N, Cwikel-Hamzany S, Matar MA, Kaplan Z, et al. High dose hydrocortisone immediately after trauma may alter the trajectory of PTSD: interplay between clinical and animal studies. Eur Neuropsychopharmacol. 2011;21:796-809.

15. Cohen H, Matar MA, Buskila D, Kaplan Z, Zohar J. Early post-stressor intervention with high-dose corticosterone attenuates posttraumatic stress response in an animal model of posttraumatic stress disorder. Biol Psychiatry. 2008;64:708-17.

16. Cohen H, Zohar J, Gidron Y, Matar MA, Belkind D, Loewenthal U, et al. Blunted HPA axis response to stress influences susceptibility to posttraumatic stress response in rats. Biol Psychiatry. 2006;59:1208-18.

17. Collimore KC, Carleton RN, Hofmann SG, Asmundson GJG. Posttraumatic stress and social anxiety: the interaction of traumatic events and interpersonal fears. Depress Anxiety. 2010;27:1017-26.

18. Christianson JP, Thompson BM, Watkins LR, Maier SF. Medial prefrontal cortical activation modulates the impact of controllable and uncontrollable stressor exposure on a social exploration test of anxiety in the rat. Stress. 2009;12:445-50.

19. Varela JA, Wang J, Christianson JP, Maier SF, Cooper DC. Control over stress, but not stress per se increases prefrontal cortical pyramidal neuron excitability. J Neurosci. 2012;32:12848-53.

20. Chakraborty $P$, Chattarji S. Timing is everything: differential effects of chronic stress on fear extinction. Psychopharmacology (Berl). 2019;236:73-86. https://doi. org/10.1007/s00213-018-5053-y.

21. Paxinos G, Watson C. The rat brain in stereotaxic coordinates: compact sixth edition. New York: Academic Press; 2009.

22. Walf AA, Frye CA. The use of the elevated plus maze as an assay of anxietyrelated behavior in rodents. Nat Protoc. 2007;2:322-8.

23. Madan JS, Gupta K, Chattarji S, Bhattacharya A. Hippocampal and amygdalar cellspecific translation is similar soon after stress but diverge over time. Hippocampus. 2018;28:441-52.

24. Schelling G, Stoll $C$, Haller M, Kapfhammer HP, Rothenhausler HB, Krauseneck T, et al. The effect of hydrocortisone during septic shock on post-traumatic stress disorder (Ptsd) in survivors. Anesthesiology. 2003;89:429A.

25. Noto $Y$, Sato $T$, Kudo $M$, Kurata $K$, Hirota $K$. The relationship between salivary biomarkers and state-trait anxiety inventory score under mental arithmetic stress: a pilot study. Anesth Analg. 2005;101:1873-6.

26. Takai N, Yamaguchi M, Aragaki T, Eto K, Uchihashi K, Nishikawa Y. Effect of psychological stress on the salivary cortisol and amylase levels in healthy young adults. Arch Oral Biol. 2004;49:963-8.

27. Armario A, Marti $O$, Molina T, De Pablo J, Valdes M. Acute stress markers in humans: response of plasma glucose, cortisol and prolactin to two examinations differing in the anxiety they provoke. Psychoneuroendocrinology. 1996;21:17-24.

28. Aerni A, Traber R, Hock C, Roozendaal B, Schelling G, Papassotiropoulos A, et al. Low-dose cortisol for symptoms of posttraumatic stress disorder. Am J Psychiatry. 2004;161:1488-90.

29. Soravia LM, Heinrichs M, Aerni A, Maroni C, Schelling G, Ehlert $U$, et al. Glucocorticoids reduce phobic fear in humans. Proc Natl Acad Sci. 2006;103:5585-90.
30. Yasmin F, Colangeli R, Morena M, Filipski S, van der Stelt M, Pittman QJ, et al. Stress-induced modulation of endocannabinoid signaling leads to delayed strengthening of synaptic connectivity in the amygdala. Proc Natl Acad Sci. 2020;117:650-5.

31. Cohen S, Kozlovsky N, Matar MA, Kaplan Z, Zohar J, Cohen H. Post-exposure sleep deprivation facilitates correctly timed interactions between glucocorticoid and adrenergic systems, which attenuate traumatic stress responses. Neuropsychopharmacology. 2012;37:2388-404.

32. Zuckerman A, Ram O, Ifergane G, Matar MA, Kaplan Z, Hoffman JR, et al. Role of endogenous and exogenous corticosterone on behavioral and cognitive responses to low-pressure blast wave exposure. J Neurotrauma. 2018; 36:380-94.

33. Jia $M$, Smerin SE, Zhang L, Xing G, Li X, Benedek D, et al. Corticosterone mitigates the stress response in an animal model of PTSD. J Psychiatr Res. 2015;60:29-39.

34. Daskalakis NP, Lehrner A, Yehuda R. Endocrine aspects of post-traumatic stress disorder and implications for diagnosis and treatment. Endocrinol Metab Clin North Am. 2013;42:503-13.

35. Yehuda R, Hoge CW, McFarlane AC, Vermetten E, Lanius RA, Nievergelt CM, et al. Post-traumatic stress disorder. Nat Rev Dis Prim. 2015;1:1-22.

36. Yehuda R, Bierer LM, Pratchett LC, Lehrner A, Koch EC, Manen JAVan, et al. Cortisol augmentation of a psychological treatment for warfighters with posttraumatic stress disorder: Randomized trial showing improved treatment retention and outcome. Psychoneuroendocrinology. 2015;51:589-97.

37. Mitra R, Sapolsky RM. Acute corticosterone treatment is sufficient to induce anxiety and amygdaloid dendritic hypertrophy. Proc Natl Acad Sci. 2008;105:5573-8.

38. Kim H, Yi J, Choi K, Hong S, Shin K, Kang S. Regional differences in acute corticosterone-induced dendritic remodeling in the rat brain and their behavioral consequences. BMC Neurosci. 2014;15:65.

39. Liston, C, Gan, WB. Glucocorticoids are critical regulators of dendritic spine development and plasticity in vivo. Proc Natl Acad Sci. 2011;108:16074-9.

40. Rajkumar RP, Bharadwaj B. Pharmacological prevention of posttraumatic stress disorder: a systematic review. Adv Psychiatry. 2014;2014:10.

41. Ludäscher $P$, Schmahl $C$, Feldmann RE, Kleindienst N, Schneider $M$, Bohus $M$. No evidence for differential dose effects of hydrocortisone on intrusive memories in female patients with complex post-traumatic stress disorder - a randomized, doubleblind, placebo-controlled, crossover study. J Psychopharmacol. 2015;29: 1077-84.

42. Astill Wright L, Sijbrandij M, Sinnerton R, Lewis C, Roberts NP, Bisson Jl. Pharmacological prevention and early treatment of post-traumatic stress disorder and acute stress disorder: a systematic review and meta-analysis. Transl Psychiatry. 2019;9:1-10.

43. Ashokan A, Sivasubramanian M, Mitra R. Seeding Stress Resilience through Inoculation. Neural Plast. 2016;1-6.

44. Brockhurst J, Cheleuitte-Nieves C, Buckmaster CL, Schatzberg AF, Lyons DM. Stress inoculation modeled in mice. Transl Psychiatry. 2015;5:1-5.

45. Lyons DM, Parker KJ, Katz M, Schatzberg AF. Developmental cascades linking stress inoculation, arousal regulation, and resilience. Front Behav Neurosci. 2009;3:1-6.

46. Hourani LL, Kizakevich PN, Hubal R, Spira J, Strange LB, Holiday DB, et al. Predeployment stress inoculation training for primary prevention of combat-related stress disorders. J Cybertherapy Rehabil. 2011:4:101-16.

47. Galatzer-Levy IR, Andero R, Sawamura T, Jovanovic T, Papini S, Ressler KJ, et al. A cross species study of heterogeneity in fear extinction learning in relation to FKBP5 variation and expression: Implications for the acute treatment of posttraumatic stress disorder. Neuropharmacology. 2017;116:188-95.

48. Sawamura T, Klengel T, Armario A, Jovanovic T, Norrholm SD, Ressler KJ, et al. Dexamethasone treatment leads to enhanced fear extinction and dynamic Fkbp5 regulation in amygdala. Neuropsychopharmacology. 2016;41:832-46.

49. Inoue R, Abdou K, Hayashi-Tanaka A, Muramatsu SI, Mino K, Inokuchi K, et al. Glucocorticoid receptor-mediated amygdalar metaplasticity underlies adaptive modulation of fear memory by stress. Elife. 2018;7:1-18.

50. Karst H, Berger S, Erdmann G, Schutz G, Joels M. Metaplasticity of amygdalar responses to the stress hormone corticosterone. Proc Natl Acad Sci. 2010;107:14449-54.

51. Joëls $M$, Sarabdjitsingh RA, Karst $H$. Unraveling the time domains of corticosteroid hormone influences on brain activity: rapid, slow, and chronic modes. Pharmacol Rev. 2012;64:901-38. 